\title{
BMJ Open Quality Reducing emergency department visits in patients with deep vein thrombosis: introducing a standardised outpatient treatment pathway
}

\author{
Tony Wan (D) , ${ }^{1}$ Anna Rahmani, ${ }^{1}$ Michaela Hanakova, ${ }^{2}$ Hing Yi Wong, ${ }^{3}$ \\ Glenyth Caragata, ${ }^{4}$ Emily S Ross, ${ }^{5}$ Oluwadamilola Akinyemi ${ }^{6}$
}

To cite: Wan T, Rahmani A, Hanakova M, et al. Reducing emergency department visits in patients with deep vein thrombosis: introducing a standardised outpatient treatment pathway. BMJ Open Quality 2021;10:e001123. doi:10.1136/ bmjoq-2020-001123

- Additional supplemental material is published online only. To view, please visit the journal online (http://dx.doi.org/10. 1136/bmjoq-2020-001123).

Received 21 July 2020 Accepted 2 June 2021

D Check for updates

(C) Author(s) (or their employer(s)) 2021. Re-use permitted under CC BY-NC. No commercial re-use. See rights and permissions. Published by BMJ.

${ }^{1}$ Medicine, The University of British Columbia, Vancouver, British Columbia, Canada ${ }^{2}$ Emergency Department, Providence Health Care, Vancouver, British Columbia, Canada

${ }^{3}$ Vancouver Coastal Health Authority, Vancouver, British Columbia, Canada

${ }^{4}$ Gerontology, Simon Fraser University, Burnaby, British Columbia, Canada

${ }^{5}$ Biomedical Physiology and Kinesiology, Simon Fraser University, Burnaby, British Columbia, Canada

${ }^{6}$ Simon Fraser University, Burnaby, British Columbia, Canada

Correspondence to

Dr Tony Wan; tony.wan2@vch.ca

\section{ABSTRACT}

Deep vein thrombosis (DVT) is an acute medical condition that requires urgent diagnosis and treatment to prevent significant morbidity and mortality. Patients with DVT frequently present to the emergency department (ED) because the necessary diagnostic investigations and medical treatment for successful outpatient management are not readily accessible in the outpatient clinics. $A$ collaborative quality improvement project was undertaken to implement and evaluate a standardised outpatient treatment pathway designed to direct patients with a newly diagnosed DVT from the ultrasound department to the thrombosis clinic, where guideline-based management for DVT can be accomplished without ED visits. During the baseline period (1 February 2017 to 31 January 2019), the number of ED visits for DVT was 383 with an average of 16 visits per month. During the intervention period (1 February 2019 to 31 January 2020), the number of ED visits for DVT was 106 with an average of 8.8 visits per month. This represents almost a $50 \%$ reduction in the average ED visits during the intervention period. A standardised outpatient treatment pathway can significantly reduce the number of ED visits in patients with DVT, potentially improving patient care and reducing ED overcrowding.

\section{INTRODUCTION}

Problem

Deep vein thrombosis (DVT), a common manifestation of venous thromboembolism (VTE), is an acute medical condition that requires urgent diagnosis and treatment to prevent significant morbidity and mortality. ${ }^{1}$ Ultrasound is the diagnostic investigation of choice in patients with a clinical suspicion for DVT. ${ }^{2}$ Untreated DVT can result in acute pulmonary embolism which is associated with a mortality rate of $25 \%$ in 3 weeks, if left untreated. $^{3}$ The recommended treatment for DVT is urgent initiation of anticoagulation therapy which is effective in preventing recurrent VTE and death. ${ }^{4}$ Most patients with DVT do not require hospital admission and outpatient treatment has similar outcomes as compared to treatment in hospital. ${ }^{5}$ Outpatient treatment of DVT has greatly simplified over the last decade due to the use of direct oral anticoagulants, including rivaroxaban and apixaban, which do not require initial parenteral anticoagulation or frequent laboratory monitoring and they are as effective as the traditional but cumbersome vitamin $\mathrm{K}$ antagonists. ${ }^{67}$

Despite the effective and straightforward outpatient treatment, patients with DVT frequently present to the emergency department (ED), often at the direction of their physicians. ${ }^{8}$ At St Paul's Hospital (SPH), an academic hospital located in British Columbia, Canada, many outpatients with suspected DVT are sent to the ultrasound department for urgent diagnosis. All patients with a confirmed DVT on ultrasound are traditionally directed to the ED immediately for acute treatment. A review of the SPH ED data between 1 February 2017 and 31 January 2019 showed 383 patients visits to the ED for the treatment of DVT. Of these patients, $355(92.7 \%)$ did not require hospital admission and they received outpatient treatment at home. However, $159(41.5 \%)$ patients needed repeat ED visits within 30 days. One possible explanation for the frequent ED visits in patients with DVT is that the necessary diagnostic investigations and medical treatment for successful outpatient management are not readily accessible in the outpatient clinics.

\section{Available knowledge}

An estimated 45000 patients in Canada are affected by DVT each year and most of the cases occur in the outpatient setting. ${ }^{9}$ In the province of Alberta, the number of ED visits leading to a diagnosis of DVT was 9194 between $2012-2014 .^{10}$ In the USA, the annual number of ED visits by patients with DVT is estimated to be 123000 and this number continues to increase every year. ${ }^{8}$ In 
the European Union, the number of estimated non-fatal symptomatic DVT events in 2004 was 684019 and the majority of the patients received treatment in the ED. ${ }^{11} 12$ In a Danish study of 9863 visits to ED, DVT represented $2.3 \%$ of the presumptive diagnosis. ${ }^{13} \mathrm{ED}$ overcrowding has been a key issue in many countries and despite increased political, administrative and public awareness, the overcrowding situation continues to rise in frequency and severity. ${ }^{14}$ To meet the care needs and reduce the burden on the ED, selected hospitals and institutions have established treatment pathways to provide timely and comprehensive outpatient treatment for patients with DVT.

The Mater Misericordiae University Hospital in Dublin evaluated a treatment pathway in the ED to support outpatient management of patients with newly diagnosed DVT. One of the major barriers to implementation was reported to be the lack of resources and supported infrastructure. ${ }^{15}$ The Queen Elizabeth II Health Sciences Centre in Nova Scotia, Canada, developed a treatment pathway to improve the transition between primary and secondary care for patients with suspected DVT. The pathway reduced the waiting time in the ED and increased referrals to the hospital's DVT clinic but the impact on reducing the number of ED visits in patients with DVT was not quantified. ${ }^{16}$ Although different hospitals and institutions have established clinical resources to support outpatient management of DVT, most patients still depend on ED visits to receive part of the treatment.

\section{Rationale}

We hypothesise that patients with DVT frequently present to the SPH ED because the necessary urgent diagnostic investigations and medical treatment are not readily accessible. The existing outpatient clinics within and around SPH may not be equipped to manage patients with newly diagnosed DVT because they are limited by inadequate capacity to assess patients within hours of referral, inability to arrange urgent laboratory test or imaging and may be staffed by physicians inexperienced in the treatment of DVT.

In January 2019, SPH expanded the thrombosis clinic with the primary aim to introducing a standardised outpatient treatment pathway that would immediately direct patients with newly diagnosed DVT from the ultrasound department to the thrombosis clinic rather than to the ED for expedited treatment. The clinic's hours of operation were increased from 4 half days a week to 10 half days a week. A full-time nursing staff was hired to support the two specialist physicians in the clinic. The part-time medical clerk was increased to full-time.

In addition to the thrombosis clinic, a reliable DVT treatment pathway is essential to direct patients to the clinic where they can access the necessary clinic resources in a timely fashion. This treatment pathway will require major changes in the workflow of multiple departments including the ED and the ultrasound department and it must be practical for all the healthcare workers involved. A collaborative quality improvement project was undertaken to implement and evaluate a standardised outpatient treatment pathway designed to direct patients with a newly diagnosed DVT from the ultrasound department to the thrombosis clinic, where guideline-based management for DVT can be accomplished.

\section{Aim}

We aimed to reduce the number of ED visits for DVT by $25 \%$ over 1 year by introducing a standardised outpatient treatment pathway that directs patients with newly diagnosed DVT from the ultrasound department to the thrombosis clinic immediately after the completion of the diagnostic ultrasound study.

\section{METHOD}

Context

SPH is a 435-bed acute care hospital affiliated with The University of British Columbia. The ED sees approximately 85000 patient visits per year. The SPH ultrasound department accepts referrals from ED and outpatient clinics within and outside of the hospital. The SPH thrombosis clinic underwent a major expansion in late January 2019 in anticipation of the implementation of the DVT treatment pathway and it now has the capacity to consistently assess urgent patient referrals on the same day. Prior to the expansion, the wait-time for urgent referrals was approximately 1-2 weeks.

\section{Intervention}

This is a collaborative quality improvement project between the ED, ultrasound department and the thrombosis clinic which is part of the Department of Medicine. This multidisciplinary team consists of physicians, nurse practitioners, nurses, sonographers, booking clerks and hospital administrators. The project aligns with the hospital and the health authority's strategic priority to reduce $\mathrm{ED}$ overcrowding by strengthening the outpatient clinic services.

Analysis of the patient journey revealed that the ultrasound department sent all patients with a newly diagnosed DVT on ultrasound to the ED for acute treatment, instead of directing the patients back to the physician who initially requested the ultrasound study. Moreover, access to diagnostic ultrasound in the ED is limited in the evening so patients who present after hours with suspected DVT, either by themselves or at the direction of their family physician, require a second ED visit the following morning, after the ultrasound is completed. Patients with a confirmed DVT on ultrasound are typically initiated on treatment by the emergency physician and then discharged home.

Since the flow of the patients is usually from the ultrasound department to the ED, the primary goal of our intervention is to establish a standardised treatment pathway that directs patients with a newly diagnosed DVT from the ultrasound department immediately to the thrombosis clinic, instead of the ED. The success of the intervention depends on a change in the workflow of the 
ED and ultrasound department so they will consistently instruct patients to go to the thrombosis clinic immediately after the completion of the diagnostic ultrasound.

\section{Study of interventions}

The interventions were tested and modified through plan, do, study, act (PDSA) cycles to implement and optimise the DVT treatment pathway. Feedback from healthcare workers in the ultrasound department and ED, who were directly involved in the care of patients with DVT, was collected by a paper survey. In addition, the physicians in the thrombosis clinic, the physician champions and administrators within the ultrasound department and ED met periodically to gather feedback. The feedback was used to identify issues and change ideas.

\section{Measures}

The primary outcome measure was the number of ED visits for DVT, defined by a primary diagnosis of lower or upper extremity DVT given by the ED physician. The diagnosis was recorded for each patient visit in the hospital's electronic medical record system. This measure was chosen because it is an important outcome for the health authorities, hospital administrators and individual patients. The ED visit data were collected systematically by the hospital administration using the existing ED database. We retrieved the data from 1 February 2017 to 31 January 2020. The baseline period is from 1 February 2017 to 31 January 2019 and the intervention period is from 1 February 2019 to 31 January 2020.

The primary process measure is the number of patients referred to the thrombosis clinic via the DVT treatment pathway. Patients referred via the pathway were identified by having a diagnostic ultrasound study performed at SPH on the same day as their initial appointment at the thrombosis clinic.

Three balancing measures include the number of patients with repeat ED visits for any reason within 30 days, the number of patients requiring admission for inpatient treatment and the length of stay of the ED visit.

The primary outcome measures and the balancing measures are collected systemically by the hospital administration using the existing ED database, which is considered reliable and consistent. The process measure is collected by a physician at the thrombosis clinic using the clinic's electronic medical record system. The same physician managed the collection in the clinic throughout the project to minimise variation.

\section{Analysis}

Two time periods were identified for analysis. The 2-year baseline period is from 1 February 2017 to 31 January 2019 and corresponds to the period of time prior to the introduction of the DVT treatment pathway. The 1-year intervention period is from 1 February 2019 to 31 January 2020 and represents the period during which the DVT pathway was developed and optimised.
Analysis of administrative data included calculation of means and proportions. Data are presented as run charts to illustrate trends over time.

This study follows the Standards for Quality Improvement Reporting Excellence 2.0 publication guidelines for reporting. ${ }^{17}$

\section{RESULT}

PDSA cycle \#1

The DVT treatment pathway was designed collaboratively by a team of thrombosis physicians, ED physicians, ED nurse practitioners, ultrasound radiologists, ultrasound sonographers and hospital administrators. One major area of improvement identified by the ultrasound radiologists and ultrasound sonographers was that patients sent in for urgent ultrasound by family physicians or walk-in clinics and then found to have a positive ultrasound had no places to go other than the ED to receive urgent treatment. The family physicians who requested the ultrasound were either unavailable to reassess the patient urgently, unable to access urgent laboratory investigations that are required before the initiation of DVT treatment, unable to access anticoagulants in a timely fashion in a primary care clinic or uncomfortable in initiating DVT treatment without the guidance from a specialist. The policy of the ultrasound department was to send these patients with a positive ultrasound to the ED, while the patients with a negative ultrasound are sent back to the family physicians. In order to avoid sending patients with a positive ultrasound to the ED, a successful DVT treatment pathway would need to allow the ultrasound department to reliably send patients to the thrombosis clinic for urgent treatment. As a result, the thrombosis clinic expanded and changed the hours of operation to Monday to Friday 08:30 to $15: 30$ to match the hours of the ultrasound department. The thrombosis clinic does not have the capacity to open weekend clinics so these patients will still be sent to the ED if they have the ultrasound on Saturday, Sunday or statutory holidays. Fortunately, the ultrasound radiologists reported that it is rare to receive urgent ultrasound requests from family physicians on the weekends or statutory holidays.

Another area of improvement identified by the ED physicians and ED nurse practitioners was the lack of access to urgent ultrasound after 16:00 when the ultrasound sonographers have gone home. As a result, the ED workflow for patients presenting with suspected DVT after hours was to arrange an ultrasound appointment the follow day and then bring the patient back to the ED for a second visit to review the result. The ED physicians reported that these patients were often unsatisfied with their care because they had to wait for hours for medical care 2 days in a row. The ideal solution is to have an ultrasound sonographer available after hours but unfortunately it is not feasible given the limited resources. Our team believed that it would be more efficient use of healthcare resource and better patient care for the second follow-up visit after the 

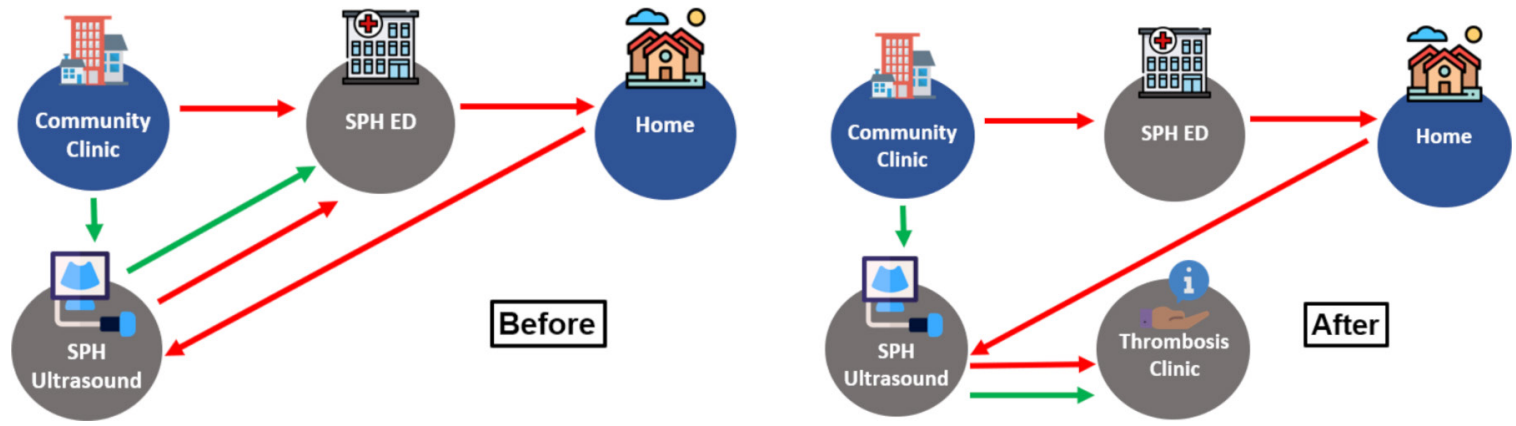

Figure 1 Patient journey before and after the introduction of the deep vein thrombosis treatment pathway. ED, emergency department; SPH, St Paul's Hospital.

ultrasound to take place in the thrombosis clinic instead of in the ED. Therefore, an important element of the DVT treatment pathway is to reliably direct these patients to the thrombosis clinic immediately after the completion of the ultrasound. The patients with a positive ultrasound will clearly benefit from assessment at the thrombosis clinic but the patients with a negative ultrasound will not require the specific expertise of the clinic. The care for patients with a negative ultrasound can be provided by family physicians but unfortunately the inner city population in our centre is frequently marginalised and many of them do not have access to primary care. As a result, the DVT treatment pathway will also direct the patients with a negative ultrasound to the thrombosis clinic to review the results and ensure that they have the appropriate follow-up care.

These two areas of improvement identified by our team aligned with an important priority of our centre as the hospital administrators work towards reducing ED overcrowding by strengthening outpatient clinic services. The support from the hospital leadership was critical to the expansion of the thrombosis clinic, which was a prerequisite to the implementation of the DVT treatment pathway.

On 28 January 2019, the DVT treatment pathway was implemented (figure 1). The pathway was communicated via email to all the involved healthcare workers including physicians, nurses, ultrasound sonographers and booking clerks by physician champions in the respective departments (online supplemental materials 1-3). Following the introduction of the pathway, the project manager arranged periodic meetings between the thrombosis clinic physicians, the ED physicians and the ultrasound department supervisor to gather feedback.

\section{PDSA cycle \#2}

Feedback from ED and ultrasound department revealed that many healthcare workers involved did not fully understand the DVT treatment pathway. Since the previous communication strategies were ineffective, information sessions were developed to provide clarification on the pathway and to promote its use to all the healthcare workers who are involved in the journey of patients with DVT. Information sessions were delivered by the thrombosis clinic physicians on 12 February 2019 at the ultrasound department noon round and on 1 June 2019 at the ED grand round. Additional feedback from all the healthcare workers in the ultrasound department and ED was collected by a paper survey. The number of patients referred to the thrombosis clinic via the pathway (primary process measure) was used to assess adherence to the pathway in the ultrasound department and ED.

\section{PDSA cycle \#3}

SPH launched a new comprehensive electronic medical record system on 17 November 2019. Although our team was aware of the plan to change the hospital's electronic medical record system, we did not know the exact date or the detail of the transition process until a few weeks prior. All communication, including referral letters to the thrombosis clinic, became electronic. Given that the ED traditionally communicated with the ultrasound department and thrombosis clinic via fax, we had to create a new referral workflow in the electronic record system. We worked with the Clinical and Systems Transformation team to create an ED DVT order plan and referral function. The design of the electronic order plan and referral function incorporated feedback from physicians and medical clerks in the ED.

\section{Outcome measure}

Results of the primary outcome measure analysis are presented as run charts to illustrate changes before and after the introduction of the DVT treatment pathway (figure 2). During the baseline period (1 February 2017 to 31 January 2019), there were 383 ED visits for DVT with an average of 16 visits per month. During the intervention period (1 February 2019 to 31 January 2020), the number of ED visits for DVT was 106 with an average 8.8 visits per month. This represents almost a $50 \%$ reduction in the average $\mathrm{ED}$ visits during the intervention period.

\section{Process measure}

The total number of patients referred to the thrombosis clinic via the DVT treatment pathway during the 1-year intervention period was 162 (figure 3). Of these patients, $67(41.4 \%)$ patients avoided all visits to the ED because they were sent directly to the ultrasound department by a physician in the community and from there to the 


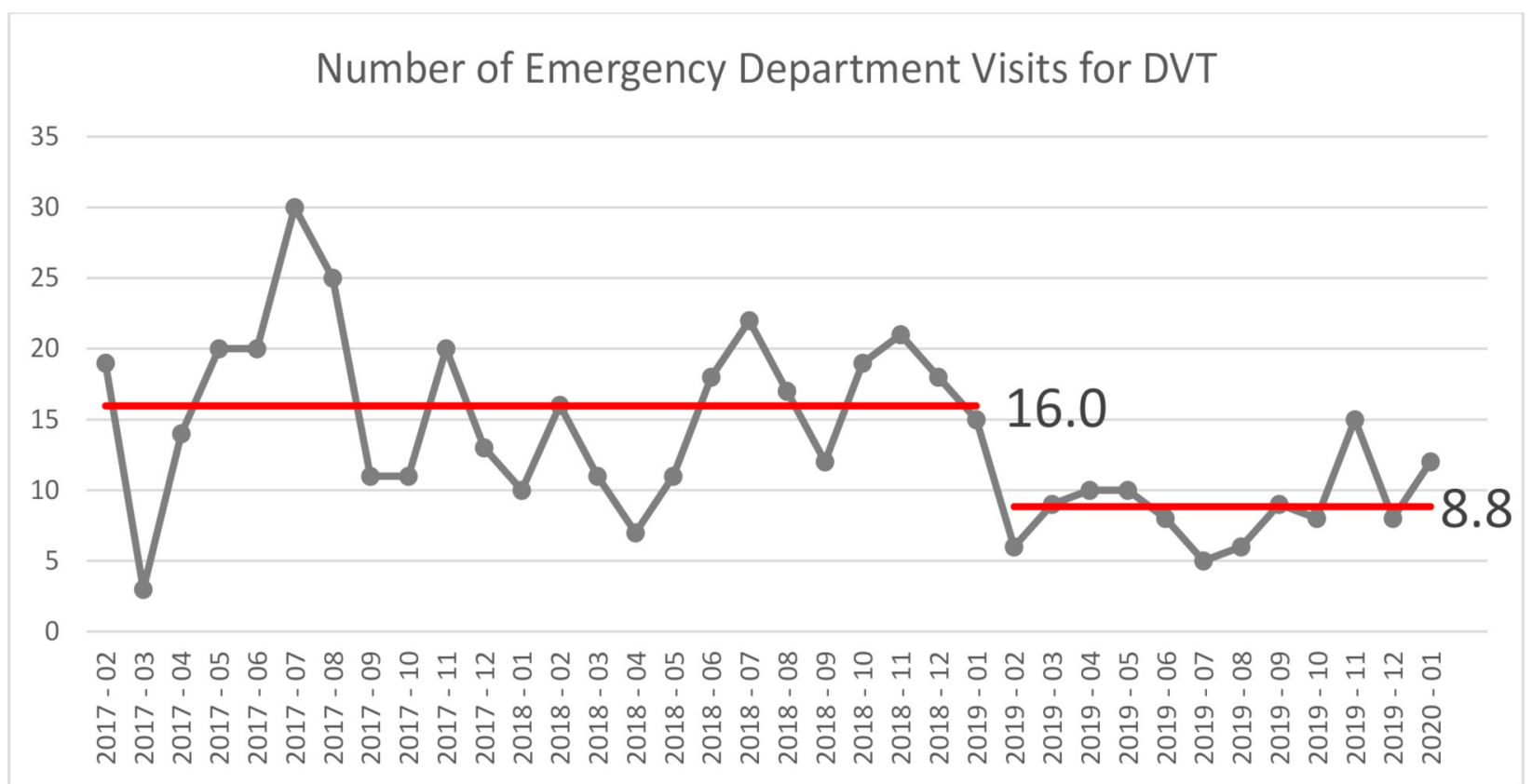

Figure 2 Monthly number of ED visits for DVT. The red lines indicate the mean number of ED visit during the baseline period and intervention period. ED, emergency department; DVT, deep vein thrombosis.

thrombosis clinic immediately after the completion of the diagnostic ultrasound (green arrows). On the other hand, $95(58.6 \%)$ patients went to the ED after hours, either on their own or at the direction of their physician, to access an urgent diagnostic ultrasound. These patients were then discharged from ED with an appointment at the ultrasound department and thrombosis clinic the following day, thus avoiding a repeat ED visit (red arrows).

\section{Balancing measures}

During the baseline period, the proportion of patients requiring repeat ED visits within 30 days was $41.5 \%$. During the intervention period, the proportion of patients requiring repeat ED visits within 30 days was $10.4 \%$. The proportion of patients who required hospital admission for the treatment of DVT was $7.3 \%$ during the baseline period and $8.5 \%$ during the intervention period. The average number of patients admitted per month was 1.2 during the baseline period and 0.8 during the intervention period. The average ED length of stay was 4.1 hours during the baseline period and 4.4 hours during the post intervention period.

\section{DISCUSSION}

Our result showed that a standardised DVT treatment pathway supported by an established thrombosis clinic was associated with a $50 \%$ reduction in the average monthly number of ED visits for DVT at SPH over 1 year. This reduction can be attributed to the fact that 162 patients were sent directly to the thrombosis clinic, instead of the ED, after the completion of diagnostic ultrasound.

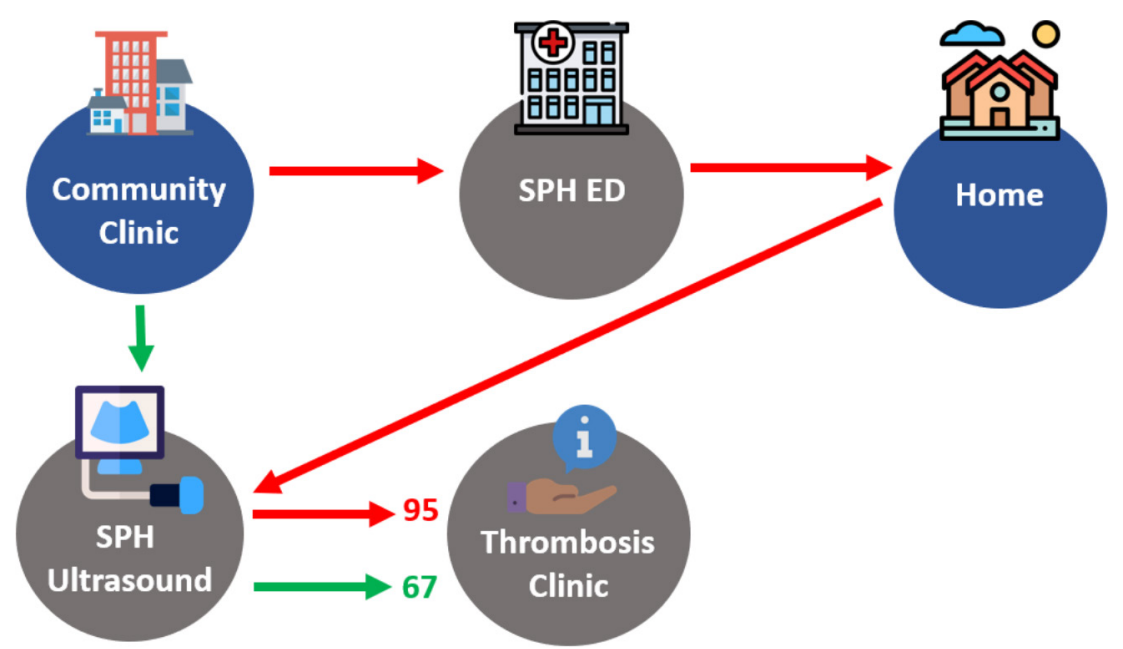

Figure 3 Number of patients referred to the thrombosis clinic via the deep vein thrombosis pathway. ED, emergency department; SPH, St Paul's Hospital. 
The magnitude of reduction in the ED visits exceeded our target of $25 \%$ reduction, likely because we underestimated the baseline number of patients who were sent by the ultrasound department to the ED for expedited treatment of DVT.

One of our balancing measures is the number of patients with newly diagnosed DVT who required repeat ED visits within 30 days. This number could be expected to increase if the patients did not adhere to each step of the treatment pathway or if the thrombosis clinic did not have the capacity to provide all necessary treatment and follow-up, requiring the patients to return to ED for additional medical interventions. However, patients requiring repeat ED visits decreased significantly from $41.5 \%$ to $10.4 \%$ during the intervention period. The prevented repeat $\mathrm{ED}$ visits were an additional benefit not completely accounted in the reported $50 \%$ reduction in ED visits for DVT because patients with DVT may return to ED for other related reasons, such as minor bleeding complications from the anticoagulation therapy, which is usually not captured under the diagnostic code of 'DVT' in the hospital's ED database. We believe that the DVT treatment pathway provided patients with a reliable follow-up and close monitoring at the thrombosis clinic, which was a care gap previously filled by ED. Patients who presented to the ED after hours when diagnostic ultrasound is not available typically needed a repeat ED visit the following day to complete and then review the diagnostic investigations. However, this second visit now happens in the thrombosis clinic instead of the ED. Additionally, selected patients with DVT, such as those with significant renal impairment or extremes of body weights, are treated with parenteral anticoagulation with transition to vitamin $\mathrm{K}$ antagonist. ${ }^{18}$ This treatment strategy requires multiple medical visits for international normalized ratio (INR) monitoring and low molecular weight heparin subcutaneous injection. With the DVT treatment pathway, these patients are now seen and followed at the thrombosis clinic, instead of the ED.

The proportion of patients requiring hospital admission for DVT and the length of stay at the ED remained consistent. This supports that the DVT treatment pathway did not introduce significant detrimental changes to the system and the patient's journey.

Several notable challenges deserve further discussion. Communication was by far the greatest obstacle to the reliable operation of the DVT treatment pathway. Multiple healthcare workers from three different departments are involved in the workflow of the pathway and it is essential for each individual to understand the roles of other providers. The physician champions from each department were instrumental to disseminating information to people within their respective departments. In addition, our hospital transitioned into a new electronic medical record system and our communication strategies had to adapt to the integrated, fully paperless, medical record system. The number of ED visits for DVT did increase during the time of transition when all the healthcare workers in the hospital were adjusting to the new system. The efficiency of the DVT treatment pathway depends on the capacity of the thrombosis clinic. As the utility of the DVT pathway increases, adequate examination rooms, physicians and support staff are important for the sustainability. This requires continual support from the hospital administrators.

We have identified a few opportunities for improvement in the future. First, some patients with DVT still required hospital admission for inpatient treatment. A closer investigation into the reasons for admission can provide insight on potential strategies to reduce the hospital length of stay or prevent the hospital admission altogether. Second, a few patients continue to require repeat ED visits despite the DVT treatment pathway. It is important to understand whether these patients returned to the ED for the management of DVT or other unrelated medical issues. Third, 95 patients during the intervention period required an ED visit to access urgent ultrasound and we would like to identify ways to improve access to urgent ultrasound without an ED visit. During data retrieval and analysis, we discovered that a group of patients visiting the ED was coded as 'rule out DVT' in the hospital ED database. The number of ED visit for 'rule out DVT' has not changed as expected as our current DVT treatment pathway does not change the prevalence of patients presenting with DVT symptoms. We suspect that these patients coded as 'rule out DVT' were sent to the ED by their family physicians or walk-in clinic for an urgent diagnostic ultrasound. It is essential to collaborate with family physicians in the community to better understand this problem and identify the barriers to sending patients directly to the ultrasound department instead of the ED.

Our project has limitations. First, this is a single-centre project and the findings will not apply to hospitals with different set-ups and resources. However, other academic centres across the world have successfully established a pathway and protocol to support the outpatient management of DVT. Second, the duration of the project was only 1 year and we will need more time and data to demonstrate the sustainability of the DVT treatment pathway. Third, our DVT pathway does not operate on weekends and statuary holidays. Our analysis did not assess the impact for patients presenting to the ED during those times. Fourth, although our results showed significant reduction in ED visit by patients with DVT, the true beneficial impact on the healthcare system is unclear without a formal cost saving analysis.

\section{CONCLUSION}

A standardised outpatient treatment pathway can significantly reduce the number of ED visits for patients with DVT, potentially improving patient care and reducing ED overcrowding. 
Acknowledgements This project was supported by the Specialist Services Committee through the Physician Led Quality Improvement initiative. The Canadian Evaluation Society contributed to the project design and data analysis.

Contributors TW and AR contributed to the design of the project, acquisition and analysis of the data and drafting of the manuscript. $\mathrm{MH}$ contributed to the design of the project and drafting of the manuscript. HYW, GC, ESR and OA contributed to the design of the project, analysis of the data and drafting of the manuscript.

Funding The authors have not declared a specific grant for this research from any funding agency in the public, commercial or not-for-profit sectors.

Competing interests None declared.

Patient consent for publication Not required.

Ethics approval Under article 2.5 of the Tri-Council Policy Statement: Ethical Conduct for Research involving Humans (TCPS2), the overarching ethical framework for research involving human participants in Canada, quality improvement activities are exempt from Research Ethics Board review. Nevertheless, we used A Project Ethics Community Consensus Initiative (ARECCI) guideline to assess the ethical implication of our quality improvement project. The project is considered minimal risk according to the ARECCI ethics guideline tool.

Provenance and peer review Not commissioned; externally peer reviewed.

Data availability statement All data relevant to the study are included in the article or uploaded as supplementary information.

Supplemental material This content has been supplied by the author(s). It has not been vetted by BMJ Publishing Group Limited (BMJ) and may not have been peer-reviewed. Any opinions or recommendations discussed are solely those of the author(s) and are not endorsed by BMJ. BMJ disclaims all liability and responsibility arising from any reliance placed on the content. Where the content includes any translated material, BMJ does not warrant the accuracy and reliability of the translations (including but not limited to local regulations, clinical guidelines, terminology, drug names and drug dosages), and is not responsible for any error and/or omissions arising from translation and adaptation or otherwise.

Open access This is an open access article distributed in accordance with the Creative Commons Attribution Non Commercial (CC BY-NC 4.0) license, which permits others to distribute, remix, adapt, build upon this work non-commercially, and license their derivative works on different terms, provided the original work is properly cited, appropriate credit is given, any changes made indicated, and the use is non-commercial. See: http://creativecommons.org/licenses/by-nc/4.0/.

ORCID iD

Tony Wan http://orcid.org/0000-0002-9820-1446

\section{REFERENCES}

1 Kearon C, Akl EA, Comerota AJ, et al. Antithrombotic therapy for VTE disease: antithrombotic therapy and prevention of thrombosis, 9th
Ed: American College of chest physicians evidence-based clinical practice guidelines. Chest 2012;141:e419S-96.

2 Lim W, Le Gal G, Bates SM, et al. American Society of hematology 2018 guidelines for management of venous thromboembolism: diagnosis of venous thromboembolism. Blood Adv 2018;2:3226-56.

3 Barritt DW, JORDAN SC. Anticoagulant drugs in the treatment of pulmonary embolism. A controlled trial. Lancet 1960;1:1309-12.

4 Carrier M, Le Gal G, Wells PS, et al. Systematic review: case-fatality rates of recurrent venous thromboembolism and major bleeding events among patients treated for venous thromboembolism. Ann Intern Med 2010;152:578-89.

5 Lozano F, Trujillo-Santos J, Barrón M, et al. Home versus in-hospital treatment of outpatients with acute deep venous thrombosis of the lower limbs. J Vasc Surg 2014;59:1362-7.

6 , Bauersachs R, Berkowitz SD, et al, EINSTEIN Investigators. Oral rivaroxaban for symptomatic venous thromboembolism. N Engl $J$ Med 2010;363:2499-510.

7 Agnelli G, Buller HR, Cohen A, et al. Oral apixaban for the treatment of acute venous thromboembolism. N Engl J Med 2013;369:799-808.

8 Yusuf H, Tsai J, Siddiqi A-E-A, et al. Emergency department visits by patients with venous thromboembolism, 1998-2009. J Hosp Adm 2012;1:1-8.

9 Kahn SR, Springmann V, Schulman S, et al. Management and adherence to VTE treatment guidelines in a national prospective cohort study in the Canadian outpatient setting. The recovery study. Thromb Haemost 2012;108:493-8.

10 Alberta Health Services. Provincial clinic knowledge topic. deep vein thrombosis, adult. emergency department, 2015Sep. Available: https://extranet.ahsnet.ca/teams/policydocuments/1/klink/et-klinkckv-deep-vein-thrombosis-adult.pdf

11 Raskob GE, Angchaisuksiri P, Blanco AN, et al. Thrombosis: a major contributor to global disease burden. Arterioscler Thromb Vasc Biol 2014;34:2363-71.

12 Jimenez S, Ruiz-Artacho P, Merlo M, et al. Risk profile, management, and outcomes of patients with venous thromboembolism attended in Spanish emergency departments: the ESPHERIA registry. Medicine 2017; $96:$ :e8796

13 Carter-Storch R, Olsen UF, Mogensen CB. Admissions to emergency department may be classified into specific complaint categories. Dan Med J 2014;61:A4802.

14 Affleck A, Parks P, Drummond A, et al. Emergency department overcrowding and access block. CJEM 2013;15:359-70.

15 Kelliher S, Hall P, Kevane B, et al. Implementation of an acute DVT ambulatory care pathway in a large urban centre: current challenges and future opportunities. Thromb J 2019;17:14.

16 Campbell S, MacDonald M, Carr B, et al. Bridging the gap between primary and secondary care: use of a clinical pathway for the investigation and management of deep vein thrombosis. $J$ Health Serv Res Policy 2008;13 Suppl 1:15-19.

17 Ogrinc G, Davies L, Goodman D, et al. SQUIRE 2.0 (standards for quality improvement reporting excellence): revised publication guidelines from a detailed consensus process. BMJ Qual Saf 2016;25:986-92.

18 Roisin Bet al. Treatment of venous thromboembolism in special populations with direct oral anticoagulants. Thrombo Haemost 2020;120:899-911. 